\title{
Controversies and Recent Advances in Hematopoietic Cell Transplantation for Follicular Non-Hodgkin Lymphoma
}

\author{
Abraham S. Kanate, ${ }^{1}$ Mohamed A. Kharfan-Dabaja, ${ }^{2}$ and Mehdi Hamadani ${ }^{1,3}$ \\ ${ }^{1}$ Myeloma and Lymphoma Service, Osborn Hematopoietic Malignancy and Transplantation Program, West Virginia University, \\ Morgantown, WV 26506, USA \\ ${ }^{2}$ Blood and Marrow Transplantation, Moffitt Cancer Center, Tampa, FL 33612, USA \\ ${ }^{3}$ Division of Hematology and Oncology, West Virginia University, P.O. Box 9162, 1 Medical Center Drive, \\ Morgantown, WV 26506, USA
}

Correspondence should be addressed to Mehdi Hamadani, mehdi.hamadani@gmail.com

Received 30 July 2012; Revised 19 September 2012; Accepted 19 September 2012

Academic Editor: Joseph H. Antin

Copyright (c) 2012 Abraham S. Kanate et al. This is an open access article distributed under the Creative Commons Attribution License, which permits unrestricted use, distribution, and reproduction in any medium, provided the original work is properly cited.

\begin{abstract}
Commonly designated as an indolent non-Hodgkin lymphoma, follicular lymphoma (FL) presents with striking pathobiological and clinical heterogeneity. Initial management strategies for FL have evolved to involve combination chemoimmunotherapy and/or radio-immunoconjugates. Unfortunately even with the best available nontransplant treatment, which nowadays results in higher frequency of response, FL remains incurable. Although considered a feasible therapeutic option, the use of hematopoietic cell transplantation (HCT) remains controversial. The appropriate timing, graft source, and intensity of HCT conditioning regimens in FL are often matters of debate. Herein we review the available published data pertaining to the use of autologous or allogeneic HCT in patients with FL across different stages of the disease, discuss major recent advances in the field, and highlight avenues for future research. The current literature does not support a role of HCT for FL in first remission, but in the relapsed setting autologous HCT remains appropriate for patients with early chemosensitive relapses, while allogeneic transplantation remains the sole curative modality for this disease, in relatively younger patients without significant comorbidities.
\end{abstract}

\section{Introduction}

Follicular lymphoma (FL) is the second most common type of non-Hodgkin lymphoma (NHL) in the western hemisphere accounting for $22 \%$ of all cases [1]. The median age at diagnosis is generally in the 6th decade, with a slight female preponderance. Being an indolent lymphoma, the disease course of FL is one of remissions and relapses with conventional chemoimmunotherapies followed not infrequently by development of resistance and/or transformation into a more aggressive histology. A subset of FL patients has a more aggressive clinical course, with approximately $15 \%$ mortality at 2 years resulting from progressive or transformed disease [2]. While clinical prognostic systems such as FL international prognostic index (FLIPI) are good in estimating overall survival (OS) [3, 4], they have limited predictive value in identifying patient groups that may (or may not) benefit from aggressive initial therapy.
Management strategies include surveillance, combination chemoimmunotherapy, radio-immunotherapy, and autologous or allogeneic hematopoietic cell transplantation (HCT). The addition of rituximab to conventional chemotherapy regimens has resulted in improved progression-free survival (PFS) and OS [5-7] in several studies.

Despite improved outcomes achieved with incorporation of monoclonal antibodies, namely, rituximab, or introduction of radio-immunoconjugates, namely, iodine I-131 tositumomab or ibritumomab tiuxetan, FL remains incurable. The role and timing of HCT in the management of FL is a controversial issue. While high-dose therapy (HDT) and autologous HCT (auto-HCT) has low treatment-related mortality (TRM) and morbidity, disease relapse remains a major concern. Myeloablative (MA) allogeneic HCT (alloHCT) is a potentially curative modality; however, it is often associated with prohibitive TRM, particularly in more frail patients. Factors to be considered while assessing patients' 
eligibility for HCT include but are not limited to patientand disease-related characteristics, optimal timing of HCT, type of HCT (autologous versus allogeneic), and selecting intensity of preparative regimens (MA or reduced-intensity conditioning (RIC)) in case an allograft is pursued $[8,9]$.

Herein we review the available published data pertaining to the role and optimal timing of HCT in patients with FL. To identify relevant publications, PubMed and Medline (the Web sites developed by the National Center of Biotechnology Information at the National Library of Medicine of the NIH), were searched using the search terms "follicular lymphoma" and "transplantation" limited to "English language," and a publication date of 1992 or later. In addition to the online database search, a manual search of the reference lists of reviews and included articles was conducted. Papers that did not include FL patients or the ones that included fewer than $25 \mathrm{FL}$ patients were excluded. Also excluded were editorials, letters to the editor, reviews, consensus conference papers, practice guidelines, and laboratory studies with no clinical correlates. National or international meetings' abstracts (American Society of Hematology, American Society of Blood and Marrow Transplantation, American Society of Clinical Oncology, European Hematology Association, and European Group for Blood and Marrow Transplantation) from January 2010 onwards and http://www.clinicaltrials.gov/ were searched to identify important ongoing trials. The goal of the paper is to critically analyze the current data pertaining to HCT in FL, in order to provide practical recommendation about the preferred graft source, conditioning regimen intensity, optimal timing, and the role of this modality in FL.

\section{Role of Transplantation for FL in First Remission}

Several studies have explored the use of HCT as consolidation after initial chemotherapy for FL, with the ultimate goal of improving the depth of response, disease control, and possibly OS.

2.1. Autologous HCT for FL in First Remission. Single center data from Dana-Farber Cancer Institute (DFCI), demonstrating prolonged disease-free survival in approximately $40 \%$ of FL patients undergoing purged bone marrow autografts, provided preliminary evidence for auto-HCT as consolidation for FL in first remission [10].

Four-randomized-controlled trials (RCT) have evaluated the role auto-HCT as consolidation for $\mathrm{FL}$ in first remission (Table 1) [11-14]. One German (German Low Grade Lymphoma Study Group (GLSG)) and two French (Groupe d'Etude des Lymphomes de l'Adulte (GELA) Groupe Ouest-Est des Leucémies et Autres Maladies du Sang (GOELAMS)) cooperative group studies randomized newly diagnosed, younger ( $\leq 60$ years), advanced stage FL patients to receive consolidation with auto-HCT or interferon maintenance, after first-line chemotherapy with CHOP (cyclophosphamide, doxorubicin, vincristine, and prednisone) or CHOP-like regimens [11-13]. As shown in
Table 1, a significant PFS benefit was demonstrated in favor of auto-HCT in the GLSG and GOELAMS trials, but not in the GELA protocol. To date no OS benefit has been reported in any published study. Despite a relatively low TRM after autografting in the GLSG trial, this modality, however, was associated with a significantly higher incidence of secondary hematological malignancies $(3.8 \%$ versus $0 \%, P=0.02)$ $[11,15]$. Similarly, significantly higher frequency of second malignancies was also seen in the GOELAMS study. A major limitation of these three trials is that they were conducted in the prerituximab era, hence questioning the applicability and relevance of these results in current practice. Interestingly, the PFS of FL patients receiving rituximab-based 1st line chemoimmunotherapy in contemporary cooperative group trials is roughly similar to the PFS reported in auto-HCT arm of GELA and GLSG studies $[6,7,16]$.

To address the role of auto-HCT in upfront consolidation of FL in the rituximab era, the Gruppo Italiano Trapianto di Midollo Osseo/Intergruppo Italiano Linfomi (GITMO/IIL) trial compared chemoimmunotherapy with $\mathrm{R}-\mathrm{CHOP}$ to rituximab supplemented HDT and auto-HCT. While rates of complete remission (CR), molecular remission, and eventfree survival (EFS) were significantly better with AutoHCT, no difference in OS was seen. A trend towards more secondary myelodysplasia/acute myeloid leukemia (sMDS/ AML) was observed in the HDT arm, albeit not statistically significant $(6.6 \%$ versus $1.7 \% ; P=0.111)$. Lack of survival benefit, despite better disease control in the auto-HCT arm, is likely due to subsequent salvage of patients relapsing after R-CHOP alone with an autograft in second (or later) remission, among other reasons [17]. Two recently published meta-analyses of aforementioned clinical trials confirmed the PFS benefit with autografting of FL patients in first remission, but no benefit in OS was described $[18,19]$.

In view of recent advances in the treatment of patients with newly diagnosed FL, including strategies such as consolidation with radio-immunotherapy [20], rituximab maintenance [21], and/or rituximab retreatment [22], routine use of autologous transplantation as consolidation in first remission for patients with FL cannot be recommended, especially as the latter is associated with development of secondary malignancies without a benefit in OS.

\subsection{Allogeneic HCT for FL in First Remission. Allo-HCT} offers several advantages such as a lymphoma-free graft, and the immunologic graft-versus-lymphoma (GVL) effect mediated by alloreactive donor $\mathrm{T}$ cells. It is a potentially curative treatment modality for patients with FL, who would be otherwise incurable with conventional chemoimmunotherapy or auto-HCT. However, there are no randomized controlled data available to support allografting in chemosensitive FL patients in first remission. Limited singleinstitution data are available for allo-HCT in a small subset of high risk FL patients with primary refractory disease, despite multiple treatment attempts [23, 24]. Such high-risk FL patients with primary refractory disease can be considered for an allo-HCT, ideally within the context of a clinical trial. At our institution, refractory FL patients are offered alloHCT as part of an ongoing prospective study evaluating 


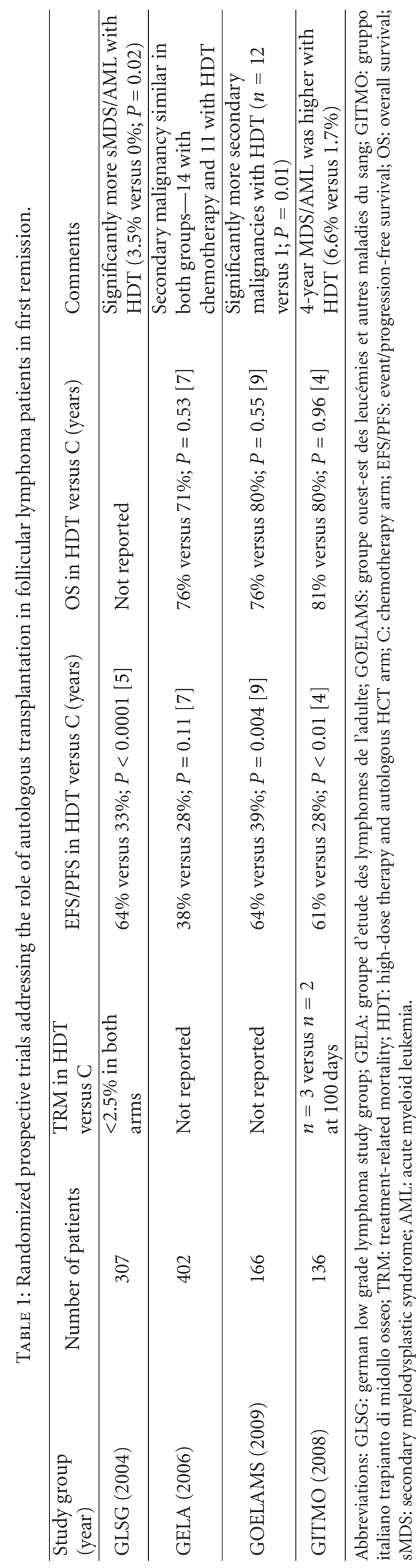


the role of pharmacokinetically guided reduced-toxicity conditioning allo-HCT for refractory aggressive lymphomas (http://www.clinicaltrials.gov/, NCT01203020).

\section{HCT for Relapsed FL}

Although the majority of FL patients respond to initial therapy, the vast majority of such patients eventually experience disease progression. HCT, autologous or allogeneic, is often considered in patients with relapsed disease, particularly after multiple lines of therapies. The role, optimal timing, and preferred transplant modality (autologous versus allogeneic) in the relapsed setting remain a matter of controversy.

\subsection{Autologous Transplantation for Relapsed FL. Auto-HCT} has long been available for patients with relapsed chemosensitive disease. Early single-institution, retrospective studies showed encouraging data for patients with relapsed disease $[25,26]$. Large prospective trials comparing auto-HCT with chemotherapy are lacking, adding to the existing controversy about the role of this treatment modality in relapsed FL. The European Blood and Marrow Transplant (EBMT) group reported the only RCT in this setting (CUP trial). The CUP trial compared chemotherapy alone to chemotherapy followed by either unpurged or purged autografts. This trial was closed early because of poor accrual. Notwithstanding the small number of patients that was randomized $(n=$ 89), the trial showed a significant PFS and OS benefit following HDT [27]. There was no reported difference in outcomes of purged compared to unpurged autografts. However, since this trial was conducted in the pre-rituximab era, its significance and clinical relevance to contemporary clinical practice is questioned.

To address the role of auto-HCT versus salvage chemotherapy alone in the rituximab-era, Sebban et al. conducted a post hoc analysis of patients enrolled on two GELF (Groupe d'Etude des Lymphomes Folliculaires) protocols that subsequently relapsed and received various salvage therapies including auto-HCT. In patients who received rituximabcontaining salvage therapies, no statistically significant EFS or OS benefits were reported after HDT and auto-HCT when compared to patients who did not undergo autografting [28]. In a different study, the combined retrospective data from DFCI and St. Bartholomew's Hospital suggested prolonged remissions in a subset of FL patients after HDT; however, this benefit appeared restricted mostly to patients in second CR [29]. Conceptually, HDT and autologous transplantation at such an early point in relapsed FL could be uniformly offered, if it was curative and devoid of long-term serious complications. Auto-HCT, unfortunately, cannot be considered a curative modality for majority of the patients with FL. Large registry data from EBMT [30] and Center for International Blood and Marrow Transplant Research (CIBMTR) show no plateau in risk of disease relapse after autografting [31]. More importantly the risks of second cancers and sMDS/AML after auto-HCT are not insignificant, ranging from 5 to $15 \%$ in several large studies $[29,30]$. While acknowledging the limitations of HDT in relapsed FL, it is also prudent to highlight the fact about a third of carefully selected chemosensitive FL patients that can experience durable responses following auto-HCT (31\% PFS at 10 years in the EBMT registry data) [30].

In order to solve the problem of autograft contamination by lymphoma cells, several studies have examined the role of ex vivo purging (by monoclonal antibodies, CD34+ cell selection, etc.) [32, 33] and in vivo purging (e.g., rituximab with mobilization) $[34,35]$ of autologous stem cell products with encouraging results. However, the lack of randomized data to prove the superiority or curative potential of purged auto-HCT [27], and a possible increase in infectious complications with ex vivo purging $[36,37]$, has prevented the uniform acceptance to this modality by transplant centers. In the rituximab era, the decision to offer an autologous transplant should take into account several factors including patient's age, associated comorbidities, risk of secondary cancers, and presence of chemosensitive disease. Heavily pretreated patients with refractory disease are unlikely to benefit from HDT and should preferably be considered for participation in clinical trials. Outside the setting of a clinical trial, the decision to offer an auto-HCT for FL should be made on a case-by-case basis. Auto-HCT is best reserved for chemosensitive, relapsed FL patients after 2-3 lines of prior chemoimmunotherapies (ideally at least one doxorubicinbased line, and a bendamustine-based regimen), who are not candidates for curative therapies, namely, allo-HCT, because of donor unavailability, associated comorbidities, or patient preference. Whether postauto-HCT rituximab maintenance will improve patient outcomes is an area of active investigation and at the moment, it cannot be considered a standard option $[38,39]$.

\subsection{Myeloablative Allogeneic Transplantation for Relapsed} FL. Adoptive immunotherapy in the form of allo-HCT is potentially curative for patients with FL. The GVL effects mediated by the donor T-lymphocytes are beneficial in patients with lymphoid malignancies $[40,41]$. One of the most compelling evidence for a clinically relevant GVL effect in relapsed FL comes from the success of allo-HCT after an autograft failure [42-44]. Unlike auto-HCT where relapserisk posttransplant does not decrease overtime, registry data from CIBMTR and EBMT $[31,45]$ clearly show that a plateau in relapse risk is achievable in 2-3 years after allografting, indicating that a substantial proportion of these patients can be cured with MA allo-HCT. However, in both CIBMTR and EBMT studies, despite impressively low relapse rates (20-25\% at 5 years) after MA allo-HCT, compared to rates following auto-HCT ( $50-55 \%$ at 5 years), no difference in OS was seen, primarily due to unacceptably high rates of TRM following MA allografts (approximately 35-40\% compared to $8-15 \%$ after auto-HCT). Moreover, since the median age at diagnosis for FL is the sixth decade of life, a significant number of such patients are not appropriate candidates for MA conditioning. Whether there is a benefit of MA allo-HCT in younger patients with chemorefractory disease, over less ablative, so-called RIC regimens, is not known. It is unlikely that a prospective clinical trial will be performed to compare MA conditioning with RIC allogeneic transplantation in 
patients with FL, as the latter has been broadly adopted as the preferred regimen to use when considering allografting. In the absence of robust prospective data to prove otherwise, MA allo-HCT should not be considered as the regimen of choice in patients with FL, especially for those with advanced age and/or with associated medical comorbidities and poor performance status.

3.3. RIC Transplantation for Relapsed FL. RIC regimens were developed to improve applicability of allo-HCT to older, heavily pretreated patients, particularly those with associated medical comorbidities. These regimens aim at reducing procedure-related toxicities and rely more heavily on GVL immunologic effects. While no prospective trials have compared MA conditioning against RIC transplantation in FL, registry data from EBMT and CIBMTR, with their inherent limitations [31,45], have established the feasibility of this approach by demonstrating acceptable rates TRM [46], albeit at the possible expense of higher relapse rates [47] and comparable OS and PFS with RIC allo-HCT compared to MA allografts.

Several phase II studies have prospectively assessed the feasibility of RIC HCT in patients with relapsed FL (Table 2) [42, 48-51]. Khouri et al. have recently reported updated M.D. Anderson Cancer Center (MDACC) experience with 47 chemosensitive FL patients conditioned with fludarabine, cyclophosphamide, and high-dose rituximab. The 11-year PFS and OS were $72 \%$ and $78 \%$, respectively. The incidence of grade $2-4$ acute GVHD was $11 \%[48,52]$. This updated report from MDACC also includes 26 patients (38\% with chemorefractory disease) who received novel conditioning with ${ }^{90} \mathrm{Y}$-ibritumomab tiuxetan. The 3-year PFS rates for patients with chemorefractory and chemosensitive disease were $80 \%$ and $87 \%$, respectively [52]. The Cancer and Leukemia Group B (CALGB) also reported encouraging outcomes of FL patients with RIC in a smaller, but multicenter prospective study [51]. The Blood and Marrow Transplant Clinical Trials Network (BMT CTN) Protocol 0701 is currently conducting a multicenter study using the RIC reported by Khouri et al. It is important to point out that the CALGB study and 2008 publication by Khouri et al. comprised almost exclusively of patients undergoing matched sibling donor HCT. To mitigate the higher rates GVHD associated with unrelated donor (URD) HCT, Thomson et al. employed in vivo T-cell depletion with alemtuzumab. In this large multicenter study, $52 \%$ of the patients underwent URD transplantation. Ten percent of cases had refractory disease. The 4-year rates of PFS, OS, and TRM were $76 \%, 76 \%$, and $15 \%$, respectively, with clinically significant acute GVHD noted in 13\% [49]. Nevertheless, relapse rates were slightly high (26\%) and donor lymphocyte infusions were frequently needed, likely because of the use of T-cell depletion.

3.4. Autologous versus RIC Allogeneic Transplant for Relapsed $F L$. A commonly encountered question in the clinic is whether to offer auto- or RIC allo-HCT to patients with FL relapsing after multiple lines of prior therapies. An adequately powered prospective trial comparing these two options is lacking [1]. Unfortunately, the very important BMT CTN 0202 trial comparing auto-HCT to RIC alloHCT in FL was closed early due to poor accrual ( $N$ for auto-HCT $=22$ and $N$ for allo-HCT $=8$ ) [53]. For the 30 patients enrolled in the BMT CTN 0202 study, the 3year OS was $73 \%$ with auto-HCT versus $100 \%$ following allo-HCT, and 3 year PFS was $63 \%$ in the auto-HCT group versus $86 \%$ in the allo-HCT cohort. No patient had grade II-IV acute GVHD. Three auto-HCT recipients died from nonrelapse causes. The Canadian group recently reported 3year PFS and OS of $96 \%$, with a novel approach of autoHCT followed by a tandem RIC allo-HCT, with low rate of TRM [54]. Whether a tandem auto/allo-HCT approach is truly superior to the current clinical practice of effective cytoreduction with chemoimmunotherapy followed by alloHCT is not known, and at the present time a tandem auto/allo-HCT should be considered investigational. While acknowledging the scarcity of good quality clinical trial data, it appears that TRM rate with RIC allo-HCT $[48,49,51]$ is relatively low, with much lower risk of disease relapse and no risk of sMDS/AML, when compared against autoHCT. Considering these facts, it is appropriate to offer RIC allo-HCT for appropriately selected and clinically fit FL patients with an available suitable adult donor, when curative intent is pursued. While the timing remains controversial, we consider this option mainly in patients who have progressed after 2-3 lines of prior therapies (including at least one with anthracyclines and/or fludarabine), provided that the disease remains chemosensitive and patients are not candidates for clinical trials. Auto-HCT can be considered for patients who are medically unfit for RIC allografting or those without an adult or alternative donor, with the understanding that cure may not be achievable.

\section{Transplantation for Transformed FL}

Histological transformation of FL (HT-FL) to aggressive NHL is not uncommon with up to $30 \%$ of FL patients undergoing transformation, at an annual rate of 3\% [55]. Studies evaluating the role of HCT in this setting are limited by a small sample size and unavailability of prospective data. Table 3 details selected studies evaluating auto-HCT for HT-FL, that involved at least 20 patients [56-60]. The EBMT reported the largest study, involving 50 patients, all with chemosensitive, disease. The 5-year PFS and OS rates were $30 \%$ and $51 \%$ respectively [61]. The Norwegian group recently published the only prospective trial of auto-HCT in HT-FL. This study showed 5-year PFS and OS rates of 32\% and $47 \%$, respectively, in 30 patients [60]. Short followup and patient selection (with majority of patients with minimal disease at transplantation) is a limitation to consider when interpreting these results. An often overlooked clinical problem in this setting is the possibility of developing late relapses, mostly involving the indolent histologic component after auto-HCT, indicating that while HDT might potentially eradicate the large cell component, the (nontransformed) FL component appears less curable in this setting.

To circumvent this problem, and to salvage patients with chemorefractory disease, limited data is available for 


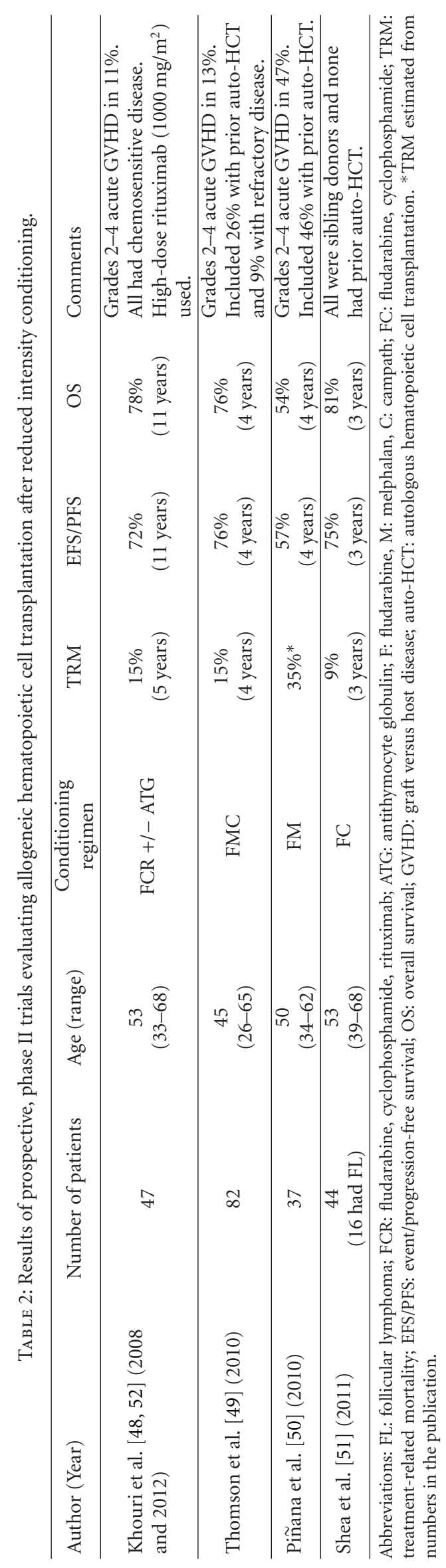




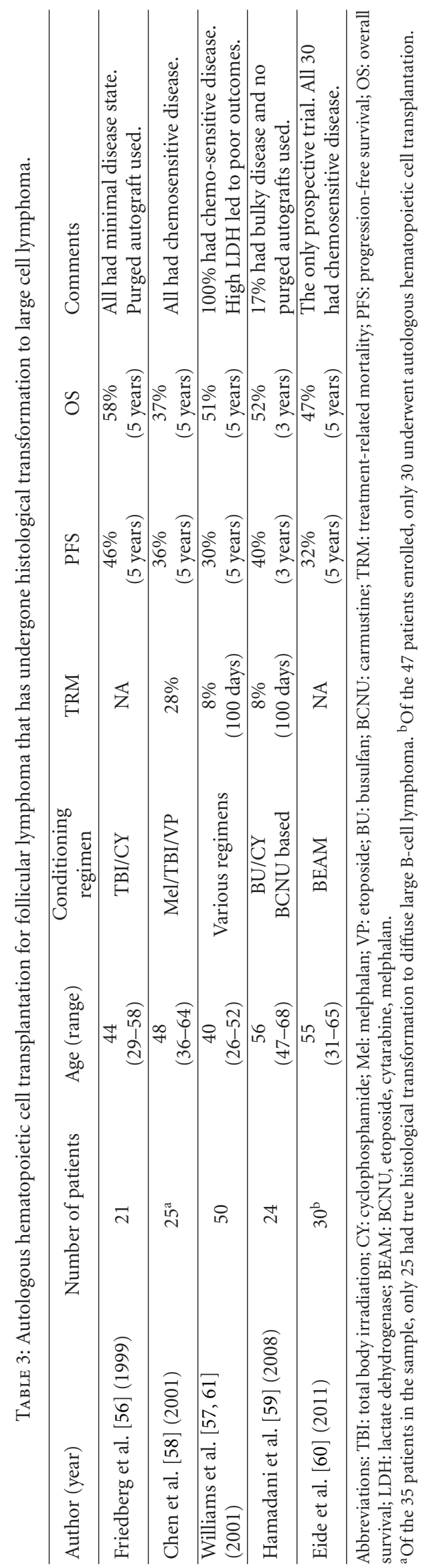


TABLE 4: Recommendations based on current evidence and expert opinion, on the role of hematopoietic cell transplantation in follicular lymphoma.

\begin{tabular}{lll}
\hline Status of FL & Type of HCT & Recommendations \\
\hline $\begin{array}{l}\text { First remission as consolidative } \\
\text { therapy }\end{array}$ & $\begin{array}{l}\text { HDT-autologous HCT } \\
\text { Allogeneic HCT }\end{array}$ & $\begin{array}{l}\text { Not recommended. } \\
\text { Not recommended. }\end{array}$ \\
\hline & HDT-autologous HCT & $\begin{array}{l}\text { Consider for patients with chemosensitive } \\
\text { disease, and } \leq 2-3 \text { lines of prior therapies. } \\
\text { Relapsed/refractory FL }\end{array}$ \\
& Myeloablative allogeneic HCT & $\begin{array}{l}\text { Best reserved for medically fit younger patients } \\
\text { with refractory disease. }\end{array}$ \\
& RIC allogeneic HCT & $\begin{array}{l}\text { Recommended for appropriately selected } \\
\text { relapsed/refractory patients. }\end{array}$ \\
\hline FL after histological transformation & HDT-autologous HCT & $\begin{array}{l}\text { Appropriate for patients with chemosensitive } \\
\text { disease. }\end{array}$ \\
& & $\begin{array}{l}\text { Ideally on a clinical trial. } \\
\text { Consider for fit patients with refractory } \\
\text { relapse, bone marrow involvement, and history } \\
\text { of prior autologous HCT. } \\
\text { Ideally on a clinical trial. }\end{array}$ \\
\hline
\end{tabular}

Abbreviations: FL: follicular lymphoma; HCT: hematopoietic cell transplantation; HDT: high-dose therapy; RIC: reduced intensity conditioning; RCT: randomized controlled trials; OS: overall survival; PFS: progression-free survival; TBI: total body irradiation; TRM: treatment-related mortality; URD: unrelated donor.

allo-HCT in HT-FL. One study evaluated the role of RIC in 16 patients with HT-FL and reported a dismal 3-year TRM, PFS, and OS rates of $43 \%, 21 \%$, and $18 \%$ respectively [62]. A South African report, which included HTFL patients $(n=11)$ who received MA conditioning, showed OS of $64 \%$ [63]. Hamadani et al. reported a $100-$ day TRM of $12 \%$ and 5-year PFS and OS of $56 \%$ and $66 \%$, respectively, in a cohort of $8 \mathrm{HT}-\mathrm{FL}$ patients that included bulky disease $(n=3)$ and/or chemorefractory disease $(n=3)$ [59]. At this time, HT-FL is best managed within the context of a clinical trial. At our institution, elderly transformed patients with minimal disease are typically offered auto-HCT, while the younger patients with minimal comorbidities and those with refractory transformed disease are offered participation on an ongoing allo-HCT clinical trial (http://www.clinicaltrials.gov/, NCT01203020).

\section{Conclusions}

Table 4 documents recommendations on the role of HCT in FL based on aforementioned reviewed data and expert opinion [64]. FL is a heterogeneous disease entity with variable presentation and clinical course. Currently, no predictive clinical or molecular markers to guide role of HCT therapy exist and this issue remains an area of active research. With improvements seen in management of newly diagnosed FL, including immunochemotherapy and rituximab maintenance [21], HCT is unlikely to play a role in the frontline setting. In the relapsed setting, prospective cooperative group effort is certainly needed to elucidate the optimal timing and overall role of HCT. Ongoing clinical trials are assessing the role of rituximab, for in vivo purging prior to auto HCT (NCT00856245) and radio-immunotherapy for disease control in the peri-transplant period. Whether the encouraging, but limited, data of tandem autologous and
RIC allo-HCT [54] in FL will play a role in future awaits confirmation with a randomized control study. For allo-HCT to become a more widely accepted curative modality for majority of FL patients it will require development of safer and less toxic conditioning regimens, more effective ways of augmenting the beneficial GVL without increasing the incidence and severity of GVHD, and improving supportive care measures after transplantation. The BMT CTN protocol 0701 (NCT00912223) is a step in the right direction, but the need remains for more robust randomized, clinical trials. While slow accrual has led to premature closure of several key clinical trials [53], continued cooperative efforts are necessary.

\section{Acknowledgments}

This work is supported in part by Conquer Cancer Foundation of ASCO Career Development Award (MH) and ASBMT New Investigator Award (MH).

\section{References}

[1] E. Ayala and M. Tomblyn, "Hematopoietic cell transplantation for lymphomas," Cancer Control, vol. 18, pp. 246-257, 2011.

[2] L. H. Sehn, T. S. Fenske, and G. G. Laport, "Follicular lymphoma: prognostic factors, conventional therapies, and hematopoietic cell transplantation," Biology of Blood and Marrow Transplantation, vol. 18, pp. S82-S91, 2012.

[3] P. Solal-Celigny, P. Roy, P. Colombat et al., "Follicular lymphoma international prognostic index," Blood, vol. 104, no. 5, pp. 1258-1265, 2004.

[4] C. Buske, E. Hoster, M. Dreyling, J. Hasford, M. Unterhalt, and W. Hiddemann, "The follicular lymphoma international prognostic index (FLIPI) separates high-risk from intermediateor low-risk patients with advanced-stage follicular lymphoma treated front-line with rituximab and the combination of 
cyclophosphamide, doxorubicin, vincristine, and prednisone (R-CHOP) with respect to treatment outcome," Blood, vol. 108, no. 5, pp. 1504-1508, 2006.

[5] M. S. Czuczman, R. Weaver, B. Alkuzweny, J. Berlfein, and A. J. Grillo-López, "Prolonged clinical and molecular remission in patients with low-grade or follicular non-Hodgkin's lymphoma treated with rituximab plus CHOP chemotherapy: 9Year follow-up," Journal of Clinical Oncology, vol. 22, no. 23, pp. 4711-4716, 2004.

[6] W. Hiddemann, M. Kneba, M. Dreyling et al., "Frontline therapy with rituximab added to the combination of cyclophosphamide, doxorubicin, vincristine, and prednisone (CHOP) significantly improves the outcome for patients with advanced-stage follicular lymphoma compared with therapy with $\mathrm{CHOP}$ alone: results of a prospective randomized study of the German Low-Grade Lymphoma Study Group," Blood, vol. 106, no. 12, pp. 3725-3732, 2005.

[7] R. Marcus, K. Imrie, P. Solal-Celigny et al., "Phase III study of R-CVP compared with cyclophosphamide, vincristine, and prednisone alone in patients with previously untreated advanced follicular lymphoma," Journal of Clinical Oncology, vol. 26, no. 28, pp. 4579-4586, 2008.

[8] M. L. Sorror, S. Giralt, B. M. Sandmaier et al., "Hematopoietic cell transplantation-specific comorbidity index as an outcome predictor for patients with acute myeloid leukemia in first remission: combined FHCRC and MDACC experiences," Blood, vol. 110, no. 13, pp. 4606-4613, 2007.

[9] M. Hamadani, M. Craig, F. T. Awan, and S. M. Devine, "How we approach patient evaluation for hematopoietic stem cell transplantation," Bone Marrow Transplantation, vol. 45, no. 8, pp. 1259-1268, 2010.

[10] J. R. Brown, Y. Feng, J. G. Gribben et al., "Long-term survival after autologous bone marrow transplantation for follicular lymphoma in first remission," Biology of Blood and Marrow Transplantation, vol. 13, no. 9, pp. 1057-1065, 2007.

[11] G. Lenz, M. Dreyling, E. Schiegnitz et al., "Myeloablative radiochemotherapy followed by autologous stem cell transplantation in first remission prolongs progression-free survival in follicular lymphoma: results of a prospective, randomized trial of the German Low-Grade Lymphoma Study Group," Blood, vol. 104, no. 9, pp. 2667-2674, 2004.

[12] C. Sebban, N. Mounier, N. Brousse et al., "Standard chemotherapy with interferon compared with CHOP followed by high-dose therapy with autologous stem cell transplantation in untreated patients with advanced follicular lymphoma: the GELF-94 randomized study from the Groupe d'Etude des Lymphomes de l'Adulte (GELA)," Blood, vol. 108, no. 8, pp. 2540-2544, 2006.

[13] E. Gyan, C. Foussard, P. Bertrand et al., "High-dose therapy followed by autologous purged stem cell transplantation and doxorubicin-based chemotherapy in patients with advanced follicular lymphoma: a randomized multicenter study by the GOELAMS with final results after a median follow-up of 9 years," Blood, vol. 113, no. 5, pp. 995-1001, 2009.

[14] M. Ladetto, F. De Marco, F. Benedetti et al., "Prospective, multicenter randomized GITMO/IIL trial comparing intensive (RHDS) versus conventional (CHOP-R) chemoimmunotherapy in high-risk follicular lymphoma at diagnosis: the superior disease control of R-HDS does not translate into an overall survival advantage," Blood, vol. 111, no. 8, pp. 4004-4013, 2008.

[15] G. Lenz, M. Dreyling, E. Schiegnitz et al., "Moderate increase of secondary hematologic malignancies after myeloablative radiochemotherapy and autologous stem-cell transplantation in patients with indolent lymphoma: results of a prospective randomized trial of the German Low Grade Lymphoma Study Group," Journal of Clinical Oncology, vol. 22, no. 24, pp. 49264933, 2004.

[16] G. Salles, N. Mounier, S. De Guibert et al., "Rituximab combined with chemotherapy and interferon in follicular lymphoma patients: results of the GELA-GOELAMS FL2000 study," Blood, vol. 112, no. 13, pp. 4824-4831, 2008.

[17] M. Ladetto, F. De Marco, F. Benedetti et al., "Prospective, multicenter randomized GITMO/IIL trial comparing intensive (RHDS) versus conventional (CHOP-R) chemoimmunotherapy in high-risk follicular lymphoma at diagnosis: the superior disease control of R-HDS does not translate into an overall survival advantage," Blood, vol. 111, no. 8, pp. 4004-4013, 2008.

[18] M. Al Khabori, J. R. de Almeida, G. H. Guyatt et al., "Autologous stem cell transplantation in follicular lymphoma: a systematic review and meta-analysis," Journal of the National Cancer Institute, vol. 104, pp. 18-28, 2012.

[19] M. Schaaf, M. Reiser, P. Borchmann, A. Engert, and N. Skoetz, "High-dose therapy with autologous stem cell transplantation versus chemotherapy or immuno-chemotherapy for follicular lymphoma in adults," Cochrane Database of Systematic Reviews, vol. 1, Article ID CD007678, 2012.

[20] F. Morschhauser, J. Radford, A. Van Hoof et al., "Phase III trial of consolidation therapy with yttrium-90-ibritumomab tiuxetan compared with no additional therapy after first remission in advanced follicular lymphoma," Journal of Clinical Oncology, vol. 26, no. 32, pp. 5156-5164, 2008.

[21] G. Salles, J. F. Seymour, F. Offner et al., "Rituximab maintenance for 2 years in patients with high tumour burden follicular lymphoma responding to rituximab plus chemotherapy (PRIMA): a phase 3, randomised controlled trial," The Lancet, vol. 377, no. 9759, pp. 42-51, 2011.

[22] B. Kahl, F. Hong, M. E. Williams et al., "Results of eastern cooperative oncology group protocol E4402 (RESORT): a randomized phase III study comparing two different rituximab dosing strategies for low tumor burden follicular lymphoma," Blood, vol. 118, LBA-6, no. 21, 2011.

[23] A. K. Gopal, K. A. Guthrie, J. Rajendran et al., " ${ }^{90}$ Y-Ibritumomab tiuxetan, fludarabine, and TBI-based nonmyeloablative allogeneic transplantation conditioning for patients with persistent high-risk B-cell lymphoma," Blood, vol. 118, no. 4, pp. 1132-1139, 2011.

[24] M. Hamadani, D. M. Benson Jr., C. C. Hofmeister et al., "Allogeneic stem cell transplantation for patients with relapsed chemorefractory aggressive non-hodgkin lymphomas," Biology of Blood and Marrow Transplantation, vol. 15, no. 5, pp. 547-553, 2009.

[25] P. J. Bierman, J. M. Vose, J. R. Anderson, M. R. Bishop, A. Kessinger, and J. O. Armitage, "High-dose therapy with autologous hematopoietic rescue for follicular low-grade nonHodgkin's lymphoma," Journal of Clinical Oncology, vol. 15, no. 2, pp. 445-450, 1997.

[26] T. M. Cao, S. J. Horning, R. S. Negrin et al., "High-dose therapy and autologous hematopoietic-cell transplantation for follicular lymphoma beyond first remission: The Stanford University experience," Biology of Blood and Marrow Transplantation, vol. 7, no. 5, pp. 294-301, 2001.

[27] H. C. Schouten, W. Qian, S. Kvaloy et al., "High-dose therapy improves progression-free survival and survival in relapsed follicular non-Hodgkin's lymphoma: results from the randomized European CUP trial," Journal of Clinical Oncology, vol. 21, no. 21, pp. 3918-3927, 2003. 
[28] C. Sebban, P. Brice, R. Delarue et al., "Impact of rituximab and/or high-dose therapy with autotransplant at time of relapse in patients with follicular lymphoma: a GELA study," Journal of Clinical Oncology, vol. 26, no. 21, pp. 3614-3620, 2008.

[29] A. Z. S. Rohatiner, L. Nadler, A. J. Davies et al., "Myeloablative therapy with autologous bone marrow transplantation for follicular lymphoma at the time of second or subsequent remission: long-term follow-up," Journal of Clinical Oncology, vol. 25, no. 18, pp. 2554-2559, 2007.

[30] S. Montoto, C. Canals, A. Z. S. Rohatiner et al., "Long-term follow-up of high-dose treatment with autologous haematopoietic progenitor cell support in 693 patients with follicular lymphoma: an EBMT registry study," Leukemia, vol. 21, no. 11, pp. 2324-2331, 2007.

[31] K. Van Besien, F. R. Loberiza, R. Bajorunaite et al., "Comparison of autologous and allogeneic hematopoietic stem cell transplantation for follicular lymphoma," Blood, vol. 102, no. 10, pp. 3521-3529, 2003.

[32] A. S. Freedman, D. Neuberg, P. Mauch et al., "Long-term follow-up of autologous bone marrow transplantation in patients with relapsed follicular lymphoma," Blood, vol. 94, no. 10, pp. 3325-3333, 1999.

[33] C. Tarella, P. Corradini, M. Astolfi et al., "Negative immunomagnetic ex vivo purging combined with high-dose chemotherapy with peripheral blood progenitor cell autograft in follicular lymphoma patients: evidence for long-term clinical and molecular remissions," Leukemia, vol. 13, no. 9, pp. 1456$1462,1999$.

[34] C. Tarella, M. Zanni, M. Magni et al., "Rituximab improves the efficacy of high-dose chemotherapy with autograft for high-risk follicular and diffuse large B-cell lymphoma: a multicenter gruppo italiano terapie innnovative nei linfomi survey," Journal of Clinical Oncology, vol. 26, no. 19, pp. 3166 3175, 2008.

[35] L. Arcaini, F. Montanari, E. P. Alessandrino et al., "Immunochemotherapy with in vivo purging and autotransplant induces long clinical and molecular remission in advanced relapsed and refractory follicular lymphoma," Annals of Oncology, vol. 19, no. 7, pp. 1331-1335, 2008.

[36] F. Crippa, L. Holmberg, R. A. Carter et al., "Infectious complications after autologous CD34-selected peripheral blood stem cell transplantation," Biology of Blood and Marrow Transplantation, vol. 8, no. 5, pp. 281-289, 2002.

[37] L. K. Hicks, A. Woods, R. Buckstein et al., "Rituximab purging and maintenance combined with auto-SCT: long-term molecular remissions and prolonged hypogammaglobulinemia in relapsed follicular lymphoma," Bone Marrow Transplantation, vol. 43, no. 9, pp. 701-708, 2009.

[38] R. Pettengell, N. Schmitz, C. Gisselbrecht et al., "Randomized study of rituximab in patients with relapsed or resistant follicular lymphoma prior to high-dose therapy as in vivo purging and to maintain remission following high-dose therapy," Journal of Clinical Oncology, vol. 28, no. 15, p. 8005, 2010.

[39] M. Magni, M. Di Nicola, C. Carlo-Stella et al., "High-dose sequential chemotherapy and in vivo rituximab-purged stem cell autografting in mantle cell lymphoma: a 10-year update of the R-HDS regimen," Bone Marrow Transplantation, vol. 43, no. 6, pp. 509-511, 2009.

[40] C. M. P. W. Mandigers, L. F. Verdonck, J. P. P. Meijerink, A. W. Dekker, A. V. M. B. Schattenberg, and J. M. M. Raemaekers, "Graft-versus-lymphoma effect of donor lymphocyte infusion in indolent lymphomas relapsed after allogeneic stem cell transplantation," Bone Marrow Transplantation, vol. 32, no. 12, pp. 1159-1163, 2003.

[41] P. Armand, H. T. Kim, V. T. Ho et al., "Allogeneic transplantation with reduced-intensity conditioning for hodgkin and non-hodgkin lymphoma: importance of histology for outcome," Biology of Blood and Marrow Transplantation, vol. 14, no. 4, pp. 418-425, 2008.

[42] I. F. Khouri, "Allogeneic stem cell transplantation in follicular lymphoma," Best Practice and Research, vol. 24, no. 2, pp. 271277, 2011.

[43] M. P. Escalón, R. E. Champlin, R. M. Saliba et al., "Nonmyeloablative allogeneic hematopoietic transplantation: a promising salvage therapy for patients with non-Hodgkin's lymphoma whose disease has failed a prior autologous transplantation," Journal of Clinical Oncology, vol. 22, no. 12, pp. 2419-2423, 2004.

[44] K. Branson, R. Chopra, P. D. Kottaridis et al., "Role of nonmyeloablative allogeneic stem-cell transplantation after failure of autologous transplantation in patients with lymphoproliferative malignancies," Journal of Clinical Oncology, vol. 20, no. 19, pp. 4022-4031, 2002.

[45] A. J. Peniket, M. C. Ruiz de Elvira, G. Taghipour et al., "An EBMT registry matched study of allogeneic stem cell transplants for lymphoma: allogeneic transplantation is associated with a lower relapse rate but a higher procedure-related mortality rate than autologous transplantation," Bone Marrow Transplantation, vol. 31, no. 8, pp. 667-678, 2003.

[46] I. Avivi, S. Montoto, C. Canals et al., "Matched unrelated donor stem cell transplant in 131 patients with follicular lymphoma: an analysis from the Lymphoma Working Party of the European Group for Blood and Marrow Transplantation," British Journal of Haematology, vol. 147, no. 5, pp. 719-728, 2009.

[47] P. Hari, J. Carreras, M. J. Zhang et al., "Allogeneic transplants in follicular lymphoma: higher risk of disease progression after reduced-intensity compared to myeloablative conditioning," Biology of Blood and Marrow Transplantation, vol. 14, no. 2, pp. 236-245, 2008.

[48] I. F. Khouri, P. McLaughlin, R. M. Saliba et al., "Eightyear experience with allogeneic stem cell transplantation for relapsed follicular lymphoma after nonmyeloablative conditioning with fludarabine, cyclophosphamide, and rituximab," Blood, vol. 111, no. 12, pp. 5530-5536, 2008.

[49] K. J. Thomson, E. C. Morris, D. Milligan et al., "T-celldepleted reduced-intensity transplantation followed by donor leukocyte infusions to promote graft-versus-lymphoma activity results in excellent long-term survival in patients with multiply relapsed follicular lymphoma," Journal of Clinical Oncology, vol. 28, no. 23, pp. 3695-3700, 2010.

[50] J. L. Piñana, R. Martino, J. Gayoso et al., "Reduced intensity conditioning HLA identical sibling donor allogeneic stem cell transplantation for patients with follicular lymphoma: long-term follow-up from two prospective multicenter trials," Haematologica, vol. 95, no. 7, pp. 1176-1182, 2010.

[51] T. Shea, J. Johnson, P. Westervelt et al., "Reduced-intensity allogeneic transplantation provides high event-free and overall survival in patients with advanced indolent B cell malignancies: CALGB 109901," Biology of Blood and Marrow Transplantation, vol. 17, pp. 1395-1403, 2011.

[52] I. F. Khouri, R. M. Saliba, W. D. Erwin et al., "Nonmyeloablative allogeneic transplantation with or without 90yttrium ibritumomab tiuxetan is potentially curative for relapsed follicular lymphoma: 12-year results," Blood, vol. 119, pp. 6373-6378, 2012. 
[53] M. R. Tomblyn, M. Ewell, C. Bredeson et al., "Autologous versus reduced-intensity allogeneic hematopoietic cell transplantation for patients with chemosensitive follicular NonHodgkin lymphoma beyond first complete response or first partial response," Biology of Blood and Marrow Transplantation, vol. 17, no. 7, pp. 1051-1057, 2011.

[54] S. Cohen, T. Kiss, S. Lachance et al., "Tandem autologousallogeneic nonmyeloablative sibling transplantation in relapsed follicular lymphoma leads to impressive progressionfree survival with minimal toxicity," Biology of Blood and Marrow Transplantation, vol. 18, pp. 951-957, 2012.

[55] A. J. Al-Tourah, K. K. Gill, M. Chhanabhai et al., "Populationbased analysis of incidence and outcome of transformed nonhodgkin's lymphoma," Journal of Clinical Oncology, vol. 26, no. 32, pp. 5165-5169, 2008.

[56] J. W. Friedberg, D. Neuberg, J. G. Gribben et al., "Autologous bone marrow transplantation after histologic transformation of indolent B cell malignancies," Biology of Blood and Marrow Transplantation, vol. 5, no. 4, pp. 262-268, 1999.

[57] C. D. Williams, C. N. Harrison, T. A. Lister et al., "High-dose therapy and autologous stem-cell support for chemosensitive transformed low-grade follicular non-Hodgkin's lymphoma: a case-matched study from the European bone marrow transplant registry," Journal of Clinical Oncology, vol. 19, no. 3, pp. 727-735, 2001.

[58] C. I. Chen, M. Crump, R. Tsang, A. Keith Stewart, and A. Keating, "Autotransplants for histologically transformed follicular non-Hodgkin's lymphoma," British Journal of Haematology, vol. 113, no. 1, pp. 202-208, 2001.

[59] M. Hamadani, D. M. Benson, T. S. Lin, P. Porcu, K. A. Blum, and S. M. Devine, "High-dose therapy and autologous stem cell transplantation for follicular lymphoma undergoing transformation to diffuse large B-cell lymphoma," European Journal of Haematology, vol. 81, no. 6, pp. 425-431, 2008.

[60] M. B. Eide, G. F. Lauritzsen, G. Kvalheim et al., "High dose chemotherapy with autologous stem cell support for patients with histologically transformed B-cell non-Hodgkin lymphomas. A Norwegian multi centre phase II study," British Journal of Haematology, vol. 152, no. 5, pp. 600-610, 2011.

[61] C. D. Williams, C. N. Harrison, T. A. Lister et al., "High-dose therapy and autologous stem-cell support for chemosensitive transformed low-grade follicular non-Hodgkin's lymphoma: a case-matched study from the European bone marrow transplant registry," Journal of Clinical Oncology, vol. 19, no. 3, pp. 727-735, 2001.

[62] A. R. Rezvani, L. Norasetthada, T. Gooley et al., "Non-myeloablative allogeneic haematopoietic cell transplantation for relapsed diffuse large B-cell lymphoma: a multicentre experience," British Journal of Haematology, vol. 143, no. 3, pp. 395403, 2008.

[63] N. Novitzky and V. Thomas, "Allogeneic stem cell transplantation with T cell-depleted grafts for lymphoproliferative malignancies," Biology of Blood and Marrow Transplantation, vol. 13, no. 1, pp. 107-115, 2007.

[64] D. M. Oliansky, L. I. Gordon, J. King et al., "The role of cytotoxic therapy with hematopoietic stem cell transplantation in the treatment of follicular lymphoma: an evidence-based review," Biology of Blood and Marrow Transplantation, vol. 16, no. 4, pp. 443-468, 2010. 


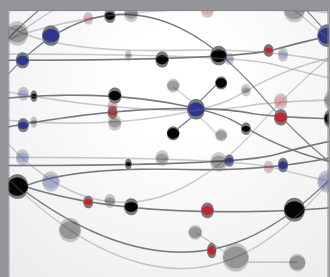

The Scientific World Journal
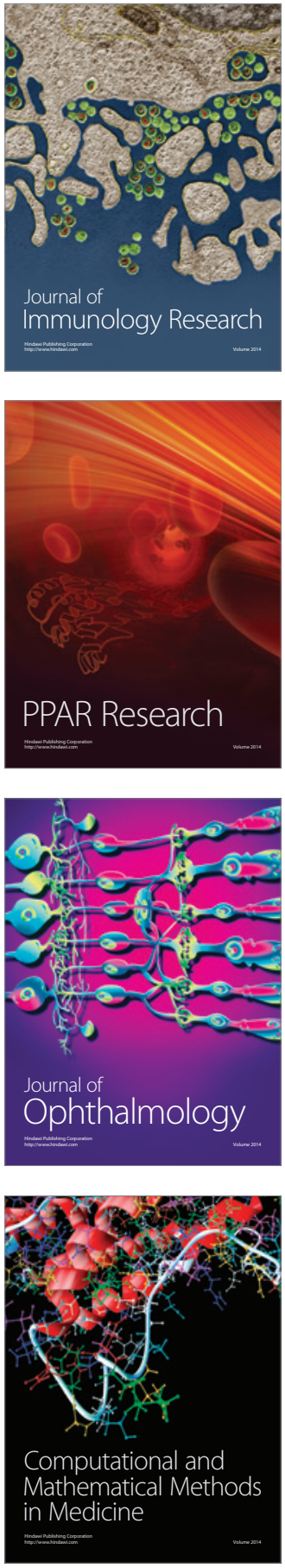

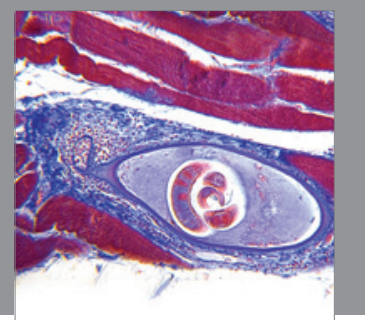

Gastroenterology

Research and Practice
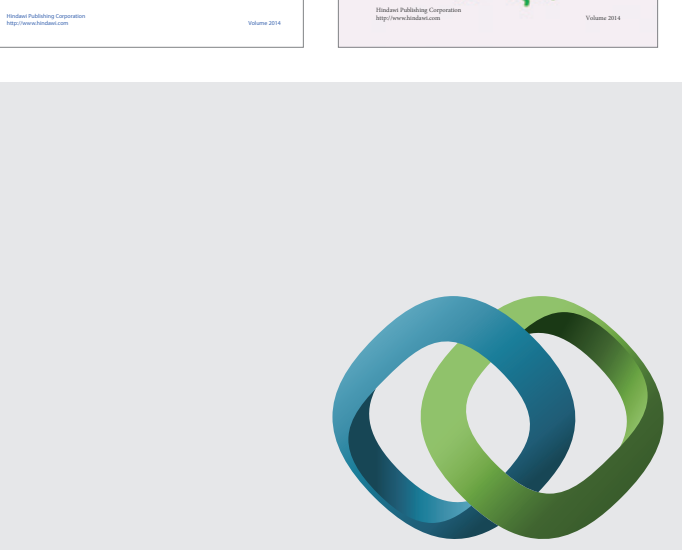

\section{Hindawi}

Submit your manuscripts at

http://www.hindawi.com
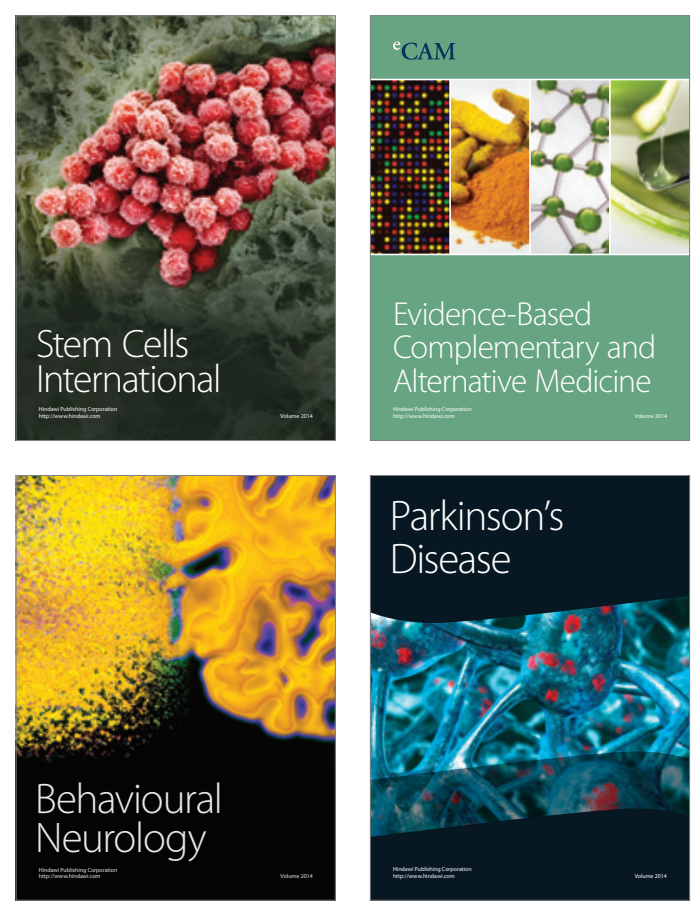

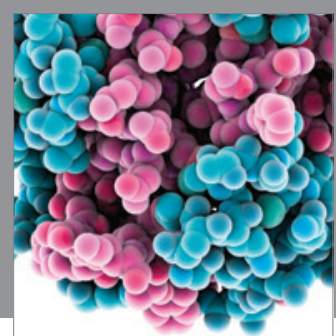

Journal of
Diabetes Research

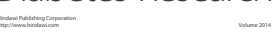

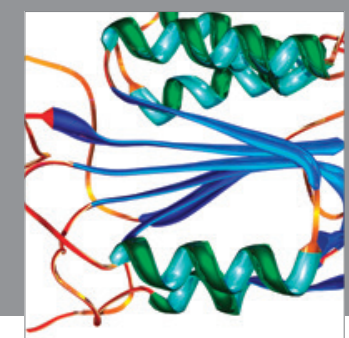

Disease Markers
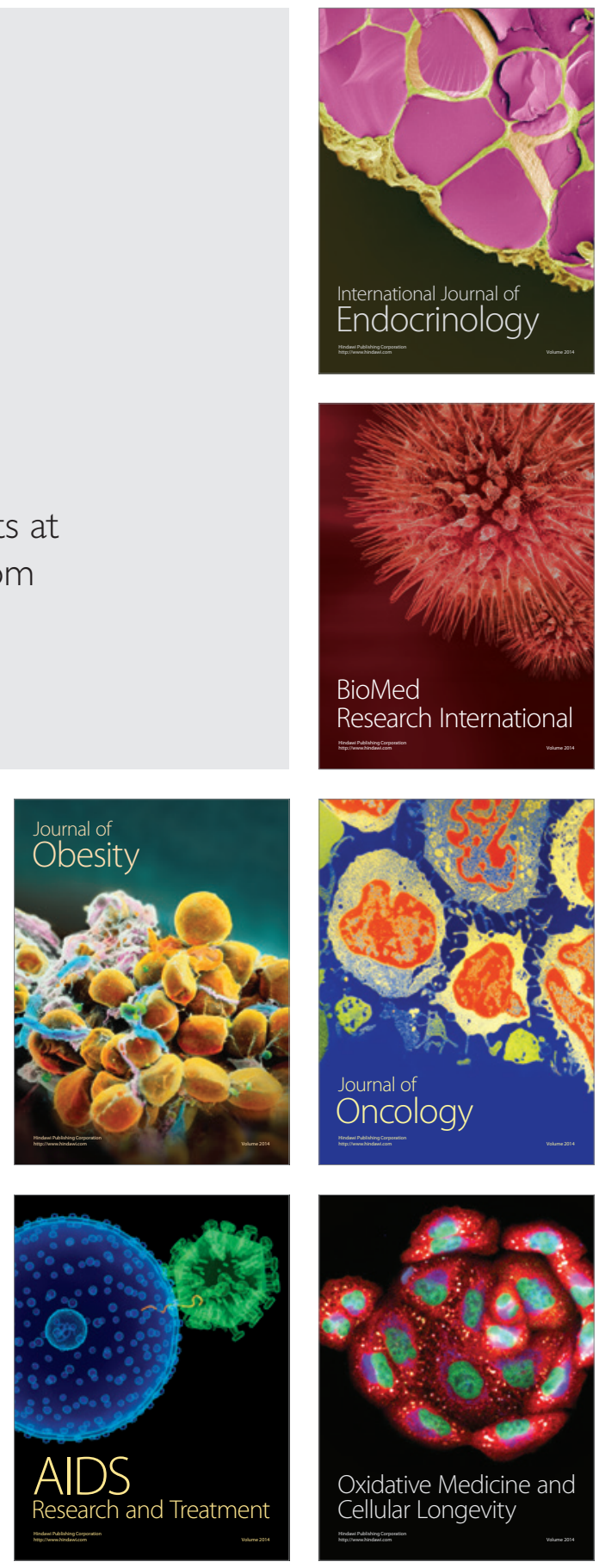